\title{
Review on foreground artificial intelligence in games
}

\author{
Rajjeshwar Ganguly ${ }^{*}$, Dubba Rithvik Reddy ${ }^{2}$, Revathi Venkataraman ${ }^{3}$, Sharanya $S^{4}$ \\ ${ }^{1,2} U G$ student, ${ }^{3}$ Professor, ${ }^{4}$ Assistant Professor \\ ${ }^{1,2,3,4}$ Computer Science and Engineering, SRM University, Chennai, India
}

\begin{abstract}
Artificial Intelligence (AI) is applied in almost every field existing in today's world and video games prove to be an excellent ground due to its responsive and intelligent behaviour. The games can be put to use model human- level AI, machine learning and scripting behaviour. This work deals with AI used in games to create more complicated and human like behaviour in the non player characters. Unlike most commercial games, games involving AI don't use the AI in the background rather it is used in the foreground to enhance player experience. An analysis of use of the AI in a number of existing games is made to identify patterns for AI in games which include decision trees, scripted behaviour and learning agents.
\end{abstract}

Keywords: Artificial Intelligence (AI), Foreground AI (F-AI), Non Playable Character (NPC), First Person Shooter (FPS), Reinforcement Learning (RL).

\section{Introduction}

In recent times artificial intelligence in games is becoming more and more intelligent which is similar to human like behavior, with the application of upcoming enhancements in the game technologies which are coming into view in game industry. Some of these techniques are used to develop AI based games to intensify player experience with the AI by foregrounding it. This project emphasizes AI use in the foreground of the game contrary to the implementation done in the background of some commercial games. Generally, an AI controller is responsible for controlling a non- player character (NPC) with which the player can intercommunicate and/or observe for a particular period of time to learn its behaviour is considered fore ground. Foreground AI can be defined as characters or objects that the player can interact and reason with. It can be deduced that AI which do not influence gameplay by interacting directly with the player is remitted to as background $\mathrm{AI}$.

The core objective of this survey is to give an insight to new types and potential genres of games. This work primarily focuses on the foreground AI in the game. The foreground AI acts as the agents, that is visible to the player and can reason about its behaviour. The Back Ground AI that is less interactive with the user and its behaviour could not be figured out. For example behavior of a NPC controlling ingame vehicle Grand Theft Auto V can be attributed to the actions of a background AI. This AI agent facilitates the player to quickly slow down on the road without frequenting crashing. The role of back ground AI should not be under estimates since they are very essential for smooth gameplay and better player experience, though players are oblivious to the actions of the back ground AI. [18]

The layout of the work is as follows: Section II reviews the various foreground AI, the importance of behaviour trees and machine learning used extensively in games. Section IV tabulates the findings from the work. Section IV limelight the open research issues. Section V discuses about the proposed system and Section VI emphasizes on the conclusion.
An overview of AI-based game design patterns and game examples. [18]

\begin{tabular}{|c|c|c|c|}
\hline Pattern & What player(s) do & Role of AI (in relation to player) & Example(s) \\
\hline Alis Visualized & Observe A Is sate & Gives (strategic) information, showing states & Third Eye Crime \\
\hline Al as Role-model & Imitate AI & $\begin{array}{l}\text { Show agent actionas and behaviors, agents as } \\
\text { puzzles }\end{array}$ & Spy Party \\
\hline Al as Trininee & Teach AI & Childstudent & Black \& White \\
\hline Al is Editable & EditAI & Artifictlagent that player can authormanipulate & Galactic Arms Race \\
\hline Al is Guided & Guidemanagge the AI & $\begin{array}{l}\text { Partly independent inhabitiants, with players as } \\
\text { their Gods }\end{array}$ & The Sims \\
\hline Al as Co-creator & $\begin{array}{l}\text { Male artificts assisted } \\
\text { by AI }\end{array}$ & Co-creator, making artifacts & ViewPoints AI \\
\hline Al as Adversary & $\begin{array}{l}\text { Play game against the } \\
\text { opponent }\end{array}$ & Opponent (symmetric) & Chess, $\mathrm{GO}_{0}$ \\
\hline Al as Villain & Combat the Villain(s) & $\begin{array}{c}\text { Villain in game, mob, boss mob, NPC } \\
\text { (asymmetic) }\end{array}$ & Alien Isolation \\
\hline Al as Spectacle & Observe & Spectacle, enacting simulated society & Nowhere \\
\hline
\end{tabular}

\section{Review}

Early games did not involve AI in their architecture. But gradually AI peeked into gaming in the form of chess, checkers, poker, bridges and many different games that out performed human intelligence or at least challenged the human intelligence. The research in games using AI advanced the computing in numerous areas. In the year 1997, the chess playing computer DEEP BLUE designed by IBM was able to win, Grandmaster Gary Kasparov in a 6 game match [1].This was a greatest milestone in gaming since the DEEP BLUE has to analyze 200,000,000 chess positions per second against a human player only who could analyze only two positions in one second. 
Jonathan Schaefferet. Al programmed implementation of a program to play checkers named CHINOOK [2] which used alpha beta pruning alongwith a transposition table, iterative deepening, search reductions, move ordering and search extensions. Scientists at the Web site of Journal Science declared CHINOOK to be absolutely unbeatable.

Michelle McPartland and Marcus Gallagher, engineered fps AI bots, that uses an autonomous learning algorithm combined with reinforcement training [3]. The work examines the results of five fps games. The designers trained the bots using training tools. The work gave a strong platform for extending the gaming AI for commercial purposes also.

Stelios Petrakis and AnastasiosTefas, educated the neural networks on the procedure of weapon or item selection in a fps game [4]. For weapon and item selection the feed forward neural networks are instructed with back-propagation. An exceptional increase in game performance could be observed.

Ronan Le Hy, Anthony Arrigoni ,Pierre Bessiere and Olivier Lebeltel, deployed Bayesian behaviour to train the video game characters[5]. The methodology maintains the state of the characters and the move decision is based on the answer to the question that involves variables and its parametric forms. This particular method however is more covenient and suitable for modeling a video game than a Finite State Machine.

Behaviour trees are hierarchies of decisions commonly used by programmers and designers in the game industry to define behaviours for agents. Ryan Marcotte and Howard J. Hamilton provide an introduction to behaviour trees [6]. Behaviour Trees provide utility in engineering or designing $\mathrm{AI}$ in games and is a plan of execution. Behavior Trees have very high utility in modeling various plans of execution and building AI in video games. Defining the characteristics of a non playable character and other such entities are carried out by behavior trees as they are perfect for the task due to their maintainability, reusability, extensibility and scalability.

Leo Galway, Darryl Keith Charles, Michaela M. Black analysis of various Machine learning techniques provides a way to enhance particularly the behavioral dynamics of artificial bots. The assistance of automated generation and the selection of various behaviours are responsible for enhancing the capabilities of video game AI and also provide the opportunity to create more engaging and entertaining game-play experiences [7].

\section{Comparative analysis}

\begin{tabular}{|c|c|c|c|}
\hline$\underline{\mathrm{S} . \mathrm{NO}}$ & Technique & year of publication & Salient Features \\
\hline 1. & Deep Blue: Computer Chess and Massively & 2002 & Demonstrates the dominance of an AI based board game. \\
\hline 2. & Solving the Game of Checkers & 1996 & Signify the use of alpha beta pruning. \\
\hline 3. & $\begin{array}{l}\text { Learning to be a Bot: Reinforcement Learning in } \\
\text { Shooter Games }\end{array}$ & 2008 & $\begin{array}{l}\text { Demonstrates how well RL can be used to learn basic FPS } \\
\text { bot behaviours. }\end{array}$ \\
\hline 4. & $\begin{array}{l}\text { Neural Networks Training for Weapon Selection } \\
\text { in First-Person Shooter Games }\end{array}$ & 2012 & Proposes weapon selection techniques in fps games \\
\hline 5. & $\begin{array}{l}\text { Teaching Bayesian Behaviours to Video Game } \\
\text { Characters }\end{array}$ & 2007 & Explores the application of Bayesian programming \\
\hline 6. & $\begin{array}{l}\text { Behaviour Trees for Modelling Artificial } \\
\text { Intelligence in Games }\end{array}$ & 2017 & $\begin{array}{l}\text { Provide an introduction the behaviour trees to strengthen } \\
\text { the AI in games }\end{array}$ \\
\hline 7. & Machine learning in digital games: A survey & 2008 & $\begin{array}{l}\text { Machine learning techniques provide a way to improve the } \\
\text { behavioural dynamics of computer controlled game agents }\end{array}$ \\
\hline
\end{tabular}

\section{Open Research Issues}

The NPCs are interacting in an unimaginable complex environment. The information retained by those environments is absurd and incomplete. Every NPC have only partial information about the state of the game. The game movement prediction can be done effectively only if the NPC is given a detailed knowledge of the state information of the game. The asymmetric nature of the games is a significant problem to frame complete state information at any instance.

From a research viewpoint the various applications of artificial intelligence in softwares such as digital games offer very interesting and unique challenges combined with several advantages [8]. This work throws a limelight on the complexity faced in the modern game domain. These complexities refer to management of resource, uncertainty decision making, spatial reasoning, temporal reasoning, modeling of opponents, collaboration and real time planning. The domain for game and AI development used several popular techniques the likes of which are Simple Decision Trees, Finite State Machines (FSM) and finally the scripting or rule based systems.

These techniques have a major limitation that the game becomes more predictable after a short amount of time. Debugging and maintaining complicated behaviors are very monotonous and tedious processes.

\section{Proposed System}

Artificial intelligence in games is mostly pre-defined to follow a set of instructions. They are scripted such that a fixed task leads to them performing a fixed action which is not the case in the system proposed. In this system the AI is entirely dynamic and can adapt to the situation and take decisions quickly depending on the given situation like a human being would. In an adaptive system the capability to predict an opponent's next move is profound. Several methods are used such as past-pattern recognition or random guessing to determine the next course of action

\section{Conclusion}

There are many areas that are being used as the testing grounds for artificial intelligence (AI) research, but games have quickly become the chosen medium and a safe place to test. Luckily, without threatening the safety and other vital systems, AI techniques in games can be tested in a game space. It is safer and better than testing it in areas where a hunchback algorithm could cost property, financial loss as well as life. The paper portrays the role of AI in gaming domain with its evolution. The various AI methodologies are discussed with their techniques and limitations. The work also high lights the open research issues that are to be addressed to move the fore ground AI to the next level. We can also infer that using behaviour trees to build AI rather than FSM's (Finite State Machines) will help to build a more complex AI that can be used to depict real life scenarios to the near accuracy. 


\section{References}

[1] URL: http://www.chessgames.com/player/deep_blue.html

[2] Jonathan Schaeffer and Robert Lake, Games of No Chance, MSRI Publications, Volume 29, 1996.

[3] Michelle McPartland Marcus Gallagher, Reinforcement learning in first person shooter games, Advances in Artificial Intelligence, 2012, 397-408.

[4] Petrakis, S., Tefas, A., Neural Networks Training for Weapon Selection in First-Person Shooter Games, Advances in Artificial Intelligence, vol. 6354, pp. 417-422. Springer.

[5] Ronan Le Hy, Anthony Arrigoni, Pierre Bessiere, Olivier Lebeltel, Teaching Bayesian Behaviours to Video Game Characters, HAL, 2007.

[6] Ryan Marcotte, Howard J. Hamilton, Behaviour Trees for Modeling Artificial Intelligence in Games: A Tutorial, the computer games journal, 2017.

[7] Leo Galway, Darryl Keith Charles, Michaela M. Black, Machine learning in digital games: A survey, 2008.

[8] Laird, J. E., van Lent, M., Human-level AI's Killer Application: Interactive Computer Games, Proceedings of AAAI 2000, pp. 1171-1178, Austin, USA, 2000

[9] Overholtzer, C.A., Levy, S.D.: Adding Smart Opponents to a First-Person Shooter Video Game through Evolutionary Design. In: Artificial Intelligence and Interactive Digital Entertainment. AAAI Press, USA (2005)

[10] [10] Sullivan, A. et al. 2012. The Design of Mismanor: Creating a Playable Quest-based Story Game. Proceedings of the International Conference on the Foundations of Digital Games (New York, NY, USA, 2012), 180-187.

[11] URL:

http://www.academia.edu/5806225/Artificial_Intelligence_in_V ideo_Games_A_Survey_Written_by

[12] URL: https://link.springer.com/chapter/10.1007/978-3-64235101-3_34

[13] URL:

http://citeseerx.ist.psu.edu/viewdoc/download?doi=10.1.1.725.3 $905 \&$ rep $=$ rep $1 \&$ type $=$ pdf

[14] Bjork, S. and Holopainen, J. 2004. Patterns in Game Design (Game Development Series). Charles River Media.

[15] Eladhari, M.P. et al. 2011. $\{\{\mathrm{AI}\}$-Based $\}$ Game Design: Enabling New Playable Experiences.

[16] Sanchez-CrespoDalmau, D.: Core Techniques and Algorithms in Game Programming. New Riders, Indianapolis (2003).

[17] van Lent, M., Laird, J. E., Buckman, J., Hartford, J., Houchard, S., Steinkraus, K. and Tedrake, R., Intelligent Agents in Computer Games, Proceedings of the National Conference on Artificial Intelligence, pp. 929-930, Orlando, USA, 1999

[18] Treanor, Mike and Zook, Alexander and Eladhari, Mirjam $\mathrm{P}$ and Togelius, Julian and Smith, Gillian and Cook, Michael and Thompson, Tommy and Magerko, Brian and Levine, John and Smith, Adam (2015) AI-based game design patterns. In: Proceedings of the 10th International Conference on the Foundations of Digital Games 2015 (FDG 2015). Society for the Advancement of Digital Games, Santa Cruz.

[19] Epic Games. (n.d.). Behaviour Trees. Retrieved June 18, 2016, from Unreal Engine Documentation: https://docs.unrealengine.com/latest/INT/Engin e/AI/BehaviourTrees/index.html

[20] Merrill, B. (2013). Building utility decisions into your existing behaviour tree. In S. Rabin (Ed.), Game AI Pro (pp. 127-136). Boston: Charles River Media.

[21] Rasmussen, J. (2016, April 27). Are behaviour trees a thing of the past? Retrieved August 2, 2016, from Gamasutra: http://www.gamasutra.com/blogs/JakobRasmussen/ 20160427/271188/Are_Behaviour_Trees_a_Thing_of_the_Past. php

[22] S.V.Manikanthan and T.Padmapriya "Recent Trends In M2m Communications In 4g Networks And Evolution Towards 5g", International Journal of Pure and Applied Mathematics, ISSN NO: 1314-3395, Vol-115, Issue -8, Sep 2017.

[23] T. Padmapriya and V. Saminadan, "Inter-cell Load Balancing Technique for Multi- class Traffic in MIMO - LTE - A Networks", International Conference on Advanced Computer Science and Information Technology, Singapore, vol.3, no.8, July 2015. 